\title{
Pavlovian temperament survey - P T S: Análise de itens e teste de realidade
}

\author{
Raquel Souza Lobo Guzzo \\ PUCCAMP \\ Isabel Cristina Riello \\ PUCCAMP \\ Ricardo Primi \\ PUCCAMP
}

\begin{abstract}
Resumo
Os estudos de personalidade e temperamento tem sido conduzidos em diferentes países buscando entender e avaliar as diferenças individuais. Esta avaliação assume importante papel no Brasil, principalmente por três razões: 1) os psicólogos no Brasil não tem suficientes pesquisas e instrumentos para avaliação psicológica; 2) não existem inventários de temperamento específicos para a prática do psicólogo na escola; 3) para a prática do psicólogo escolar a avaliação de características de personalidade é uma função importante. O objetivo deste trabalho foi estabelecer referências para o conceito e dimensões avaliadas pela versão Brasileira do PTS e promover análises para a clarificação do constructo desta área. A amostra foi composta por 395 sujeitos de diferentes grupos de idade que responderam ao instrumento. Foi conduzi da uma análise de itens. Os resultados foram discutidos considerando a avaliação internacional do PTS.

Palavras-chave: temperamento, personalidade, testes.
\end{abstract}

\section{Pavlovian Temperament Survey - P T S: iten and validity analisys}

\begin{abstract}
Summary
The studies of personality ant temperament has been conducted in different countries in order to betler understand and assess the individual differences. This issue assumes an important role in Brazil maily because of three kinds of reasons: 1) Brazilian psuchologist has no sufficient research and instruments for psychological assessment; 2) There is no specific temperament scale for school psychologist practices and 3) for school psychologist service the assessment for personality characteristics is an important function. The purpose ofthis study was to establish empirical referents for the particular concept and dimensions assessed by PTS-Brazilian version, and providing an empirical analysis for the clarification ofthe constructs ofthis area. Third hundred nithy five (395) subjects from different group age, answered the scale, and it was conducted a analysis of the items. Results was described and discussed considering the international evaluation ofthe PTS. Key words: temperament, personality, tests
\end{abstract}

A Psicologia como ciência apresenta inúmeros desafios, dentre eles o de desvelar as características individuais com procedimentos e instrumentos apropriados ao indivíduo, à sua cultura, ao contexto imediato em que vive e se desenvolve.

As questões referentes às diferenças individuais têm sido uma constante na busca do conhecimento psicológico, e tem ocorrido nas últimas décadas um crescente interesse pelos "fenômemos da individualidade temperamental e sua significância funcional para o desenvolvimento normal e desviante" (Thomas et alii, 1982:1). Dentre as características individuais, o temperamento tem sido estudado de diversas maneiras e relacionado a diversas variáveis. No entanto, tem prevalecido uma ênfase nas suas possíveis bases genéticas e suas relações com o

\footnotetext{
* Trabalho financiado pelo CNPq (processo 521625/97-7 Projeto Integrado) contou com a colaboração de 2 bolsistas de Iniciação Científica - Maria Carolina Scoz e Maria Cristina Pinho, graduação em Psicologia e Daniel Righato, aperfeiçoamento.
} 
meio-ambiente. As relações entre as bases genéticas e as influências do ambiente sobre as características individuais, principalmente de temperamento, tem suscitado interesse especialmente nas questões de ajustamento do indivíduo a suas condições de desenvolvimento (Lemer, 1990). Apesar de existirem distintas vertentes sobre essas questões, o caminho das investigações sobre esse tema geralmente buscam "novas ênfases em psicologia do desenvolvimento e em processos sociais interativos" (Goldsmith, 1990: 250).

Com objetivos variados, cada área tem usado uma concepção de temperamento em suas proposições, o que tem levado à algumas dificuldades de consenso quanto à natureza desse termo.

Mesmo tendo sido explorado desde o início do século (Heymans 1909), o temperamento mais atualmente volta como um tema importante para pesquisa sobre o funcionamento humano.

Dentre as visões atuais que tem estimulado significantes esforços de pesquisa sobre temperamento, pode-se destacar as investigações de Thomas e Chess (1982), Plomin (1982), Windle e Lemer(1984), Rothbart(1986), Goldsmith et alii (1987), Strelau (1991), Hofstee e Raad (1992).

No entanto, apesar das diferenças conceituais existentes, Strelau (1991) lembra que uma das condições para se fazer progresso em pesquisas de temperamento é mostrar um determinado instrumento de diagnóstico e sua teoria subjacente, e relacioná-la a outras mensurações e teorias de temperamento.

Algumas teorias permitem a relação entre suas definições de temperamento e praticamente todas concebem o temperamento como um fenômeno presente desde o início da vida e relativamente mais estável que muitas outras características e diferenças comportamentais (Rutter, 1987; Goldsmith, 1990, Keogh e Bess, 1990; Strelau, 1995).

A maioria dos autores citados desenvolveu, a partir de concepções teóricas específicas, instrumentos para a medida do temperamento. Essa preocupação com procedimentos e instrumentos para a avaliação de características individuais tem sido constante também entre pesquisadores brasileiros. Guzzo (1995) e Pasquali (1995) apontaram as carências de instrumentos destinados a esta prática e a necessidade urgente de desenvolvimento de pesquisas nacionais.

O temperamento, entendido como uma das características que define as diferenças individuais, pode, com seu estudo, oferecer maior conhecimento das tendências pessoais e das redes de percepções existentes entre os indivíduos (Keogh e Bess, 1990).

Estudos contemporâneos sobre esse tema tem gerado diferentes concepções teóricas na Europa e Estados Unidos (Plomin,1982; Thomas et alii,1982; Rothbart, 1986; Goldsmith, 1990, Strelau, 1991). Apesar das divergências existentes entre as principais abordagens, alguns pontos de consenso podem ser destacados, como as possíveis bases biológicas do temperamento: sua existência desde o início da vida e sua estabilidade em comparação com outras expressões emocionais. Também são considerados relevantes para a constituição da personalidade em geral, e do temperamento em especial, a influência do contexto social e a dinâmica existente entre o mesmo e as características temperamentais individuais.

Interessados na avaliação deste tema, Buss e Plomin especificam como elementos constituintes do temperamento três características principais: 1) emotividade (equivalente a reações emocionais intensas fora de controle, especificamente negativas); 2) atividade (tempo, força, ritmo 
e amplitude); e 3) sociabilidade (preferência por estar com outros). O instrumento desenvolvido para avaliar estas dimensões é o Temperament Survey (EASI, Buss and Plomin, 1975, apud Strelau, 1990).

Já Windle e Lemer (1984) propõem a avaliação do temperamento através de algumas dimensões como: aproximação-retração; flexibilidade-rigidez; nível de atividade de sono, ritmicidade, humor e persistência. Para tal avaliação desenvolveram o instrumento Revised Dimensions of Temperament Survey (DOTS-Windle e Lemer, 1986).

Reforçando a importância do instrumento como elemento primordial na investigação de características de personalidade, além dos já citados, outros estudos tem sido desenvolvidos no sentido de adaptar, validar ou criar instrumentos de mensuração (Goldsmith e Rieser-Danner, 1990; Rothbart, 1981; Strelau et alii 1991).

Algumas revisões sobre instrumentos (Hubert, 1982; Keogh e Bess, 1990) consideram que a posição da avaliação do temperamento tem melhorado sua qualidade e mostram propriedades psicométricas razoáveis, e boa confiabilidade de teste-reteste.

No entanto, este é um campo de investigação que merece muita atenção, já que a construção e/ou adaptação de escalas destinadas a todas as faixas etárias e diferentes contextos, são fundamentais e constituem o primeiro passo para qualquer trabalho de investigação do temperamento, suprindo deficiências metodológicas e fornecendo subsídios para delimitações conceituais.

Neste contexto, observa-se que no Brasil há carência de trabalhos destinados à adaptação e validação de instrumentos de mensuração. Para Oakland (1989) o Brasil ainda se encontra no estágio de utilizar instrumentos de outras culturas, muitas vezes, sem a necessária validação e adaptação para nossa realidade.

Um dos esforços neste sentido foi desenvolvido por Riello (1992), para avaliação do temperamento de adolescentes brasileiros. A autora estudou um instrumento. (SSQ- Student Styles Questionnaire, Oakland, Glutting and Horton, 1996) que avalia quatro dimensões dicotômicas do temperamento, ou seja, introversão/extroversão, sensitividade/intuição,emotividade/racionalidade, crítica/percepção. Este estudo, além de reduzir a escala original de 100 itens para 61 itens, levantou questões importantes sobre a consistência de fatores avaliadores do temperamento.

Uma concepção que tem influenciado o pensamento de pesquisadores do Leste Europeu é a Teoria Regulativa do Temperamento (Strelau, 1995). Nesta concepção o temperamento toma parte na regulação da relação entre o homem e seu mundo externo, e características como reatividade e atividade desempenham uma função de reguladoras. Para verificação destas propriedades em correlatos comportamentais, Strelau et alii (1991) elaboraram um instrumento, o PTS-Pavlovian Temperament Survey, que permite a avaliação de dimensões como Força de Excitação, Força de Inibição e Mobilidade, através da auto percepção. Este instrumento teve sua origem na cultura alemã, hoje no entanto, ampliando seu nível de investigação em um programa internacional que inclui a participação de diferentes países representando diferentes culturas e idiomas.

Visando possibilitar a avaliação de características temperamentais, esta pesquisa teve como objetivo realizar o estudo do PTS para a realidade brasileira de acordo com os seguintes passos: a) verificar o comportamento do instrumento (seus itens) nesta realidade, a consistência interna; b) chegar a uma versão brasileira do instrumento que permita a continuidade de seus estudos de 
validação e; c )através de juízes independentes, verificar a clareza dos constructos.

\section{Método}

\section{Sujeitos}

O presente estudo contou com dois grupos de sujeitos que serviram para as duas etapas de procedimentos. Para tal, num primeiro momento fizeram parte 392 sujeitos, sendo 143 do sexo masculino (36.5\%) e 249 do sexo feminino (63.5\%), em idades variando de 10 a 72 anos (média=29), conforme aparece descrito na Tabela 1. Os níveis de instrução e classes sociais foram diversos.

As informações necessárias para a caracterização dos sujeitos foram coletadas a partir do cabeçalho da folha de resposta.

Tabela 1. Distribuição dos Sujeitos segundo as variáveis sexo e idade

\begin{tabular}{|c|c|c|c|c|}
\hline Sexo & \multicolumn{2}{|c|}{ Masculino } & \multicolumn{2}{c|}{ Feminino } \\
\hline Faixa etária & f & $\mathbf{5}$ & f & \% \\
\hline 10 a 15 & 6 & 4,2 & 11 & 4,5 \\
\hline 16 a 20 & 23 & 16,1 & 73 & 29,2 \\
\hline 21 a 26 & 38 & 26,5 & 56 & 22,4 \\
\hline 27 a 35 & 38 & 24,5 & 49 & 19,7 \\
\hline 36 a 46 & 16 & 11,2 & 36 & 14,4 \\
\hline 47 a 57 & 14 & 9,8 & 9 & 3,7 \\
\hline 58 a 72 & 11 & 7,7 & 15 & 6,1 \\
\hline Total & 143 & 100,0 & 249 & 100,0 \\
\hline Válidos & 140 & - & 248 & - \\
\hline Perdidos & 3 & - & 1 & - \\
\hline Média & 30.700 & - & 28.504 & - \\
\hline DP & 12.832 & - & 12.557 & - \\
\hline
\end{tabular}

Num segundo momento contou com oito juizes, psicólogos ou estudantes de psicologia, dos quais quatro, já tinham conhecimento anterior da teoria de temperamento que embasa o instrumento, e os outro quatro, receberam informação da teoria somente por meio do material de análise, que definia os constructos envolvidos.

\section{Material}

Foi utilizado para coleta de dados o instrumento Pavlovian Temperament Survey (PTS, Strelau, Angleitner e Ruch, 1991 - anteriormente denominado STI-R - Strelau Temperament Inventary) composto por 252 itens com quatro pontos de avaliação na forma Likert. Seus itens estão divididos em três fatores baseados nas propriedades Pavlovianas do SNC: 1) Força de excitação; 2) Força de inibição e 3) Mobilidade do processo nervoso.

O instrumento é apresentado na forma de um caderno com questões e instrução para aplicação e folha de resposta, que contém um cabeçalho com informações específicas sobre os sujeitos (nome, data de nascimento, idade, sexo, para estudante a escola, série, curso, opção profissional, nível de escolaridade dos pais. Para profissional, o nível de escolaridade, profissão, local de trabalho e função exercida).

Ainda foi utilizada uma folha com descrição dos constructos a serem avaliados e de seus delimitadores, ou seja representação do constructo no comportamento, as quais foram utilizadas 
pelos juizes em suas avaliações.

\section{Procedimento}

O procedimento desse estudo constituiu-se em duas etapas distintas. A primeira na aplicação do instrumento à 392 sujeitos. A aplicação foi realizada por três psicólogos e três estudantes de psicologia devidamente treinados. Devido a variedade de idade e profissão dos sujeitos, a aplicação foi realizada em diversos locais, como escolas, ambientes de trabalho e residências, em aplicação individual e em grupo (permitida pelo formato do instrumento).

O segundo procedimento de coleta de dados destinou-se a classificação de cada item do instrumento de acordo com os constructos propostos pelos seus autores, pelos sujeitos que funcionaram como juizes, classificando cada um dos itens de acordo com sua compreensão de que os itens estavam avaliando, tendo como referência a teoria.

\section{Resultados}

Os dados coletados nesse estudo referem-se às respostas dos sujeitos ao instrumento, como forma de avaliação dos ítens do inventário. Além desses, foi feita a avaliação dos itens por juizes, de acordo com sua pertinência aos constructos definidos como fatores avaliados pelo instrumento. Os resultados desse estudo serão descritos de acordo com os objetivos propostos.

Considerando-se as respostas dos 392 sujeitos foi realizada uma análise de consistência interna computando-se a correlação item-total e os coeficientes Alfa por cada sub-escala. Esta análise permitiu identificar 19 itens considerados como mais consistentes dentro de suas respectivas subescalas (totalizando 57 itens). A partir da escala reduzida foi realizada novamente a mesma análise de correlação, apontando como cada item correlacionava-se aos respectivos fatores e o conseqüente valor alfa de cada fator separadamente. Os resultados apontam os fatores em ordem de maior valor alfa. Mobilidade $(0,83)$, Força de Excitação $(0,81)$ e Força de Inibição $(0,74)$ (Tabela 2). Os resultados apresentados demonstram que estatísticamente os fatores Força de Excitação, Força de Inibição e Mobilidade são consistentes para os itens relacionados na versão brasileira da escala. 
Tabela 2. Valor item-total dos itens e o valor alfa dos fatores

\begin{tabular}{|c|c|c|c|c|c|}
\hline & F. Excitação & & F. Inibição & & Mobilidade \\
\hline Itens & Iten-total & Itens & Iten-total & Itens & Iten-total \\
\hline 043 & 0,26 & 008 & 0,30 & 012 & 0,39 \\
\hline 049 & 0,299 & 011 & 0,34 & 018 & 0,41 \\
\hline 061 & 0,377 & 017 & 0,37 & 030 & 0,39 \\
\hline 103 & 0,26 & 170 & 0,24 & 165 & 0,38 \\
\hline 112 & 0,26 & 250 & 0,53 & 171 & 0,45 \\
\hline 136 & 0,50 & 053 & 0,22 & 036 & 0,53 \\
\hline 245 & 0,35 & 185 & 0,22 & 057 & 0,47 \\
\hline 148 & 0,51 & 101 & 0,28 & 063 & 0,46 \\
\hline 151 & 0,48 & 107 & 0,24 & 183 & 0,51 \\
\hline 169 & 0,52 & 140 & 0,28 & 189 & 0,49 \\
\hline 172 & 0,61 & 143 & 0,31 & 087 & 0,38 \\
\hline 178 & 0,39 & 152 & 0,26 & 093 & 0,51 \\
\hline 184 & 0,32 & 161 & 0,26 & 102 & 0,44 \\
\hline 196 & 0,31 & 239 & 0,32 & 105 & 0,38 \\
\hline 203 & 0,27 & 220 & 0,41 & 114 & 0,39 \\
\hline 219 & 0,30 & 231 & 0,31 & 153 & 0,22 \\
\hline 226 & 0,37 & 232 & 0,28 & 156 & 0,27 \\
\hline 230 & 0,34 & 237 & 0,34 & 150 & 0,36 \\
\hline 236 & 0,44 & 244 & 0,27 & 202 & 0,34 \\
\hline v. Alfa & 080 & v. Alfa & 0,73 & v. Alfa & 0,83 \\
\hline
\end{tabular}

Quando comparadas as variáveis entre si (sexo, idade, Força de Excitação, Força de Inibição e Mobilidade), foram encontradas diferentes correlações. Os fatores se correlacionaram mais entre si do que com outras variáveis, no entanto a idade apareceu correlacionada com o fator Força de Inibição (Tabela 3).

Tabela 3. Correlação entre as variáveis sexo, idade, força excitação, força inibição e mobilidade

\begin{tabular}{|c|c|c|c|c|c|}
\hline Variáveis & Sexo & Idade & $\begin{array}{c}\text { Força de } \\
\text { excitação }\end{array}$ & $\begin{array}{c}\text { Força de } \\
\text { inibição }\end{array}$ & $\begin{array}{c}\text { Mobili- } \\
\text { dade }\end{array}$ \\
\hline Sexo & 1.00 & & & & \\
\hline Idade & 0,08 & 1.00 & & & \\
\hline F. Excit & 0,09 & 0,01 & 1.00 & & \\
\hline F. inib & 0,08 & $0,24^{* *}$ & $0,18^{*}$ & 1.00 & \\
\hline Mobil & 0,03 & 0,04 & $0,36^{* *}$ & $0,13^{*}$ & 1.00 \\
\hline
\end{tabular}

Com a finalidade de verificar o grau de confiabilidade dos dados de classificação dos ítens por juízes, foram analizadas as respostas referentes a escala reduzida, com 57 itens. Considerando 19 itens em cada fator, os mesmos tinham 152 oportunidades de serem classificados no fator pelos 8 juízes.

As avaliações dos juizes independentes mostraram que o fator Força de Excitação foi o que apresentou maior divergência na classificação, portanto, menor clareza; Esta divergência indicou que existe maior dificuldade de identificação entre os itens dos fatores de Força de Excitação e Força de Inibição em comparação com Mobilidade, mais claramente identificado. Considerando-se o fator Força de Inibição, também pode-se observar dificuldades de compreensão entre os itens dos fatores Força de Excitação e Força de Inibição (Tabela 4). 
Tabela 4. Freqüência de classificação dos itens pelos juizes

\begin{tabular}{|c|c|c|c|c|c|}
\hline Fator & $\begin{array}{c}\text { Força } \\
\text { Excitação }\end{array}$ & Inibição & $\begin{array}{c}\text { Modali- } \\
\text { dade }\end{array}$ & Total & $\mathrm{x}^{2}$ \\
\hline F. Excit & 64 & 23 & 152 & 128 & 47 \\
\hline F.Inib & 93 & 21 & 152 & 128 & 47 \\
\hline Mobilid & 19 & 15 & 118 & 152 & 0,98 \\
\hline
\end{tabular}

$\mathrm{N} . \mathrm{gl}=2, \mathrm{P}<0,05, \mathrm{Xc}=5,99$

Os dados de concordância foram correlacionados de forma a diferenciar os juizes, em relação aos seus contatos, com a teoria. Considerando-se os dados gerais, observou-se que houve diferença entre a avaliação dos juizes que tiveram contato anterior 'com a teoria e os que não tiveram (correlação mais baixa). A correlação entre juizes quanto a concordância na classificação dos itens nos fatores foi maior entre o grupo daqueles que tiveram contato anterior com a teoria, do que os que não tiveram.

Tabela 5. Correlação entre juizes

\begin{tabular}{|c|c|c|c|c|c|c|c|c|}
\hline & 1 & 2 & 3 & 4 & 5 & 6 & 7 & 8 \\
\hline 1 & & & & & & & & \\
\hline 2 & 0,66 & & & & & & & \\
\hline 3 & 0,55 & 0,61 & & & & & & \\
\hline 4 & 0,36 & 0,48 & 0,38 & & & & & \\
\hline 5 & 0,16 & 0,20 & 0,30 & 0,52 & & & & \\
\hline 6 & 0,15 & 0,23 & 0,18 & 0,37 & 0,30 & & & \\
\hline 7 & 0,27 & 0,35 & 0,30 & 0,43 & 0,39 & 0,37 & & \\
\hline 8 & 0,34 & 0,46 & 0,45 & 0,22 & 0,22 & 0,24 & 0,21 & \\
\hline
\end{tabular}

Quando os fatores foram analisados isoladamente na comparação entre os dois grupos de juizes, pode-se observar diferenças de índice de concordância entre eles. No fator Força de Excitação a diferença de acerto foi de $74 \%$ para $11 \%$ entre os dois grupos de juizes. Em Força de Inibição a diferença foi de $77 \%$ para $45 \%$, e em Mobilidade a diferença foi um pouco menor, de 91\% para 64\%. Estes dados também apontam para o fato de que o fator Mobilidade foi o que apresenta o menor número de discordância entre os juizes. (Tabela 6)

Tabela 6. Porcentagem de concordância entre juízes e grupos de juízes

\begin{tabular}{|c|c|c|c|}
\hline Fatores & FE & FI & MO \\
\hline Juízes & $\%$ & $\%$ & $\%$ \\
\hline 1 & 63 & 68 & 89 \\
\hline 2 & 63 & 95 & 100 \\
\hline 3 & 100 & 89 & 95 \\
\hline 4 & 68 & 58 & 79 \\
\hline \% total de acertos & 74 & 77 & 91 \\
\hline 5 & 11 & 53 & 74 \\
\hline 6 & 16 & 31 & 63 \\
\hline 7 & 16 & 37 & 53 \\
\hline 8 & 0,5 & 58 & 68 \\
\hline$\%$ total de acertos & 11 & 45 & 64 \\
\hline
\end{tabular}


Parece evidente pelas análises realizadas que o fator Mobilidade está mais claramente definido e compreendido do que os fatores Força de Inibição e Força de Excitação.

\section{Discussão}

A partir dos dados apresentados alguns questionamentos podem ser levantados com respeito aos itens e fatores estudados.

A dificuldade quanto a identificação dos itens dos fatores Força de Inibição e Força de Excitação e, principalmente a dificuldade em diferenciar Força de Excitação de Força de Inibição, pode acontecer devido uma dicotomia entre esses fatores, os quais possivelmente podem ser definidos como dimensões opostas de um mesmo constructo.

Mobilidade foi o fator identificado com mais clareza pelos juizes, fato que pode estar ligado a sua maior independência em relação aos outros fatores, como a uma possível melhor elaboração de seus itens. A avaliação mais clara dos juizes neste fator também foi reforçada pela análise de correlação item/total que indicou a maior consistência interna em Mobilidade.

Quando realizadas as correlações entre os fatores, observou-se maior correlação entre Mobilidade e Força de Excitação. Este dado é reforçado pela literatura que aponta para dados semelhantes quando realizadas análises de correlação entre os mesmos fatores. Kang (1995), obteve correlação de 0,47 entre Mobilidade e Força de Excitação e 0,18 para Mobilidade e Força de Inibição, na versão coreana da PTS. Na versão americana, Clark e Newberry (1995) encontraram correlações de 0,43 entre Mobilidade e Força de Excitação enquanto entre Mobilidade e Força de Inibição a correlação encontrada foi 0,16.

A análise dos itens e fatores, além de permitir a redução do instrumento na primeira versão brasileira, permitiu a identificação de seus pontos fortes e vulneráveis que serão básicos para novas análises a serem realizadas. Esta versão permitirá a adaptação e validação futura do instrumento.

Em relação ao outro objetivo desse estudo, os dados da presente pesquisa também permitiram verificar que o contato com a teoria permite maior clareza na identificação dos itens, o que remete a necessidade de conhecimento teórico para a realização de estudos com instrumentos psicológicos. Assim, pode-se dizer que é fundamental o aprofundamento teórico e de características culturais da definição do temperamento, como padrão comportamental influenciado em grande parte pela cultura.

\section{Referências}

Goldsmith, H. H.; Buss, A. H.; Plomin, R.; Rothbart, M. K.; Chess, S.; Thomas, A.; Hindle, R.A.; Mccall, R.B. (1987). Roundtable: What is temperament? four Aproaches. Child Development, 58: 505-529.

Goldsmith, H. H. and Rieser-Danner, A. (1990). Assessing early temperament. In: Reinolds, C.R. and Kamphaus, R.W. Handbook of Psychological and Educational Assessment of Children: Personality, behavior, and context: 245-271.

Guzzo, R.S.L. (1995). Aplicação na escola das técnicas do exame psicológico. Boletim de Psicologia, 14 (102); 45-49.

Heymans, G. (1909). De Toekomistige eeuw der psychologie; rede uitgesproken bij de overdracht van het rectoraat der Rijksuniversiteit te Groninger (the future century of Psychology).

Hofstee, W.K.B. and Raad, B. de (1992). Integration of the Big Five and circumplex approaches to trait structure. Joumal of personality and social Psychology, 63 (I), 146-163.

Hubert, N.C. et alii (1982). The study of early temperament: measurement and conceptual issues Child 
Development, 53: 571-600.

Keogh, B.K.; Bess, C.R. (1990). Assessing temperament In: H.Lee Swanson. Handbook on the assessment of learning disabilities: theory, research, and practice. Austin, Texas, 313 - 329.

Oakland, T. (1989). Psicologia escolar no Brasil: passado, presente e futuro. Psicologia Teoria e Pesquisa, Brasília, 5 (2), 199-201.

Oakland, T.; Horton, C. and Glutting, J. (1996). Student Styles Questionnaire. The Psychological Corporation, Harcout Brace Company, São Antonio, Texas.

Pasquali, L. (1995). Construção de Escalas Psicológicas. Curso ministrado - Instituto de Psicologia Departamento de Pós-Graduação da PUCCAMP (Mímeo).

Plomin, R. (1982). The difficult concept of temperament : a response to Thomas, Chess and Korn. Merril Palmer - Quarterly, 28: 1-20

Riello, I. C. (1992). O temperamento de adolescentes: estudo de um instrumento de avaliação. Dissertação de Mestrado. Universidade Católica de Campinas, Campinas-SP.

Rothbart, M.K. (1981). Measurement of temperament in infancy. Child Development, 52,569-578. 365.

Rutter, M. (1987). Temperament, personality and personality disorder. British Journal of Psychiatry, 150: 443-458.

Strelau, J. (1991). Temperament and Giftdness in Children and Adolescents. Conferência apresentada na "Ninth World Conference on Gifted and Talented Children Hage - Netherland.

Strelau, J.; Willibald, R.; Angleitner, A. (1991). The Strelau Temperament Inventory Revised (STI-R): Validity Studies. European Journal of Personality, 5,287-308.

Strelau, J. (1995). The regulative theory of temperament: current status. Paper presented at the 7th Meeting of International Society for the Study of Individual Differences, Warsaw, Poland, July 15-19.

Thomas, A.; Chess, S. and Kom, S.J. (1982). The reality of difficult temperamento Merril - Palmer Quarterly, 28 (1): 1-20.

Windle, M. (1986). A fatorial replication study of the adult rvised dimensions of temperament Survey. Journal of Personality Assessment, 53 (4), 685-692.

Windle, M. and Lemer, R.m. (1984). The role of temperament in dating relationship among young adults. Merril-Palmer Quarterly, 30 (2): 163-175. 